\title{
Erratum to: Cardiac PET/MRI: Technical Requirements and Clinical Applications
}

\section{Stephan G. Nekolla • Christoph Rischpler •} Anja Batrice • Markus Schwaiger

Published online: 1 May 2013

(C) Springer Science+Business Media New York 2013

Erratum to: Curr Cardiovasc Imaging Rep (2013) 6:158-168

DOI 10.1007/s12410-013-9190-8

The title of this article has been revised to "Cardiac PET/MRI:

Technical Requirements and Clinical Applications."

The online version of the original article can be found at http://dx.doi.org/ 10.1007/s12410-013-9190-8.

S. G. Nekolla $(\varangle) \cdot$ C. Rischpler $\cdot$ M. Schwaiger

Nuklearmedizinische Klinik und Poliklinik, Technische

Universität München, Klinikum rechts der Isar,

Ismaningerstrasse 22,

81675 München, Germany

e-mail: Stephan.Nekolla@tum.de

\section{A. Batrice}

Klinikum rechts der Isar, Ismaningerstrasse 22,

81675 München, Germany 